\title{
Effect of drug urine abnormality in adolescent age group
}

\author{
Available online at www.ijistweb.com \\ RESEARCH ARTICLE \\ Aashutosh, Dinesh Kumar* \\ Department of Biochemistry, Sri Satya Sai University of Technology \& Medical Sciences, Sehore- \\ 466001 \\ *Corresponding Author’s E-mail: om11agra85@gmail.com
}

\begin{abstract}
Adolescents have $2 / 3$ of total population. This is a unique group with special needs and atention. Most adolescents with lifestyle issues fell in the age group of 16-18 years. A complete urinalysis includes physical, chemical and microscopic examinations. Midstream clean catch collection is acceptable in most situations, but the specimen should be examined within two hours of collection. Cloudy urine often is a result of precipitated phosphate crystals in alkaline urine, but pyuria also can be the cause. A strong odour may be the result of a concentrated specimen rather than a urinary tract infection. Dipstick urinalysis is convenient, but false-positive and false-negative results can occur. Specific gravity provides a reliable assessment of the patient's hydration status. Glomerular, renal and urologic causes of microhaematuria often can be differentiated by other elements of the urinalysis. Although transient proteinuria typically is a benign condition, persistent proteinuria requires further work-up. Uncomplicated urinary tract infections diagnosed by positive leukocyte esterase and nitrite tests can be treated without culture.
\end{abstract}

Keywords: Urinalysis, Life Style, Glomerular Filtration

\section{Introduction}

Adolescent population and health of adolescents is a very special issue and is focus of attention globally for various reasons. Adolescence is an important transition in an individual's life. Turmoil which one experiences in this period-has greater effects onto the behavioural aspects of an individual. If one divides the whole stream of an individual's life into certain urbitrary segments, then adolescence is that period of life which lies between childhood and adulthood (1-4). Adolescence is characterised by a strong tendency to experiment with risk behaviour.

\section{Why Test?}

Standard methods of adherence monitoring for prescribed substances, for example, selfreporting and monitoring of symptoms or patient behaviors, are unreliable for controlled substances.

\section{Whom to Test?}

Practice guidelines on pain management intended to promote safe and competent opioid prescribing recommend various measures to mitigate risk including UDT, but some disagreement persists on who should be subjected to routine UDT and its frequency.
Two types of drug testing assays are available: qualitative tests usually used for screening and quantitative tests used for confirmation. Qualitative tests are point-of-care tests and home drug test kits. They are easy to perform, relatively inexpensive, and use immunoassays, such as enzyme-linked immunoassay or radioimmunoassay, that give instant positive or negative results. Although they are sensitive and function well as screening tests, they are susceptible to cross-reactions, resulting in false-positive results, which limit their specificity. Some laboratories use qualitative tests as a "screening procedure"; in this method, negative test results are discarded, and only positive test results are run through more expensive confirmatory testing, although the 2-step procedure has become less common as the cost of confirmatory testing has decreased. Confirmatory tests are performed in laboratories and not at the point of care. Most laboratories use a combination of gas chromatography and mass spectrometry and can positively identify a substance and generate quantitative concentrations (5-7).

Urine screening can provide an indication that someone has consumed drugs at some point 
before sampling. Most drugs typically have a detection window of up to 48 hours (Table). This may be shorter for some drugs which are eliminated relatively quickly from the body such as alcohol or gamma hydroxybutyrate. Drugs such as diazepam and cannabis can persist in urine for days or even weeks and so can be detected for longer periods. The frequency of drug consumption will also have an impact on the effectiveness of urine screening. Acute or once-off use, as in the case of drug-facilitated crime where a drug is used to render someone incapacitated, is more difficult to detect and may challenge the sensitivity of urine screening. People who use drugs more regularly will typically have higher concentrations of drugs in their urine leading to easier detection with possibly longer timeframes (8-9).

In chronic users, drugs of abuse can be detected in urine for approximately one week after last use, and in extreme cases even longer in cocaine (22 days) and cannabis users (up to three months). Urine testing will not show when a drug was used, or how much. Importantly, a positive drug result cannot infer impairment at the time the urine was collected (10-12). Most commonly used drug screening tests involve immunoassay techniques (13). These range from so-called bedside or point-of-care testing to more sophisticated laboratory-based immunological tests. Immunoassay can provide fast and reliable results, however the results must be interpreted with caution.

Table 1 Advantages and disadvantages of urine used for drug testing.

\begin{tabular}{|lll|}
\hline \multicolumn{2}{|c|}{ Advantages } & \multicolumn{1}{c|}{ Disadvantages } \\
\hline - Available in sufficient quantities & - Short to intermediate window of detection \\
- Higher concentrations of parent drugs and/or & - Easy to adulterate or substitute \\
metabolites than in blood & - May require observed collection \\
- Availability of point-of-care tests (POCTs) & - Some individuals experience "shy bladder" \\
- Well-researched testing techniques & & syndrome and cannot produce a specimen \\
\hline
\end{tabular}

\section{Materials and Methods}

In hospital or pathology laboratories the emphasis is typically on drugs of abuse, screening either for compliance to (e.g. methadone) or abstinence from (e.g. cannabis) particular substances. Hospital screening can also give the clinician additional help in the differential diagnosis and treatment of a patient, but the availability of toxicological analyses varies between hospitals.

Most commonly used drug screening tests involve immunoassay techniques. These range from so-called bedside or point-of-care testing to more sophisticated laboratory-based immunological tests. Immunoassay can provide fast and reliable results, however the results must be interpreted with caution.

\section{Sample and settings}

We approached all subjects aged 12 to 18 years and Individual schools and classes were selected randomly

Forensic Urine Drug Testing
In forensic drug testing, results are meant to stand up to legal challenges and meet the rules of evidence in legal proceedings. Chain-ofcustody procedures, secure storage of samples, and stringent method validations are utilized with the aim of minimizing or eliminating false positive results, and rigorous laboratory certification programs are used to assure quality. The personnel running the tests in a forensic UDT laboratory usually have training in chemistry or forensic science and they understand chain-of-custody and medicolegal requirements.

\section{Results and Discussion}

The valid detection period for drug exposure varies depending on the disposition characteristics of the drug, dose, and frequency of use. Specific characteristics of a urine sample include its appearance, temperature within 4 minutes of voiding, $\mathrm{pH}$, creatinine concentration, and specific gravity. The color of urine is based on the concentration of its constituents and can vary based on medications, 
foods, or disease states; excess hydration can cause it to appear colorless. Concentrated urine

Table 2 Detection of drug in Urine specimens are usually more reliable than dilute specimens.

\begin{tabular}{ll}
\hline Drug or drug class & Detection times in urine \\
\hline $\begin{array}{l}\text { Benzodiazepines (e.g. alprazolam, diazepam, } \\
\text { temazepam) }\end{array}$ & $1-7$ days or longer depending on half-life of drug* \\
Cannabinoids & $3-28$ days depending on frequency of use \\
Cocaine & $1-3$ days \\
Methamphetamine/amphetamine & $2-5$ days \\
Methylenedioxymethamphetamine & $2-5$ days \\
Opioids (e.g. morphine, codeine) & $1-2$ days \\
Steroids (e.g. testosterone, stanozolol) & $\begin{array}{l}\text { Days to months depending on the half-life of the } \\
\text { steroid }\end{array}$
\end{tabular}

* may be longer in chronic users

Most drugs of interest are first detected by simple immunoassays. These are broad screening tests that are quick, often cheap and effective for showing a positive or negative result. However, as with all screening tests there are limitations to the degree of interpretation that can be inferred from the result. The limitations of immunoassay techniques include false positives as well as false negative results.

Benzodiazepines: Variability in immunoassay cross-reactivity also applies to benzodiazepines. While many benzodiazepines are generally detected by immunoassay, not all benzodiazepines are equally detectable by all reagents. Clinicians should carefully interpret the presence or absence of the benzodiazepine class when assessing treatment adherence.

In most instances it is useful to have a private conversation with the adolescent to clarify interpretation of the drug test result. Simply sharing that the drug test yielded an "unexpected result" without revealing specific details may set the stage for an honest conversation about substance use, and at times, patients will reveal use of substances that were not detected by the test. If the patient gives a history that is consistent with the drug test results the conversation can move on to a discussion of next steps - which could include changes to the treatment plan. Sharing drug test results together with a plan may facilitate a positive conversation.

When a drug test result is dilute or otherwise ambiguous a clinical interview may be helpful. Starting with a simple statement about an "unexpected test result" without revealing all of the details can serve as an openended way of beginning the conversation. If a patient does not report substance use the clinician can review methods for reducing the chance of a dilute specimen - by providing a first morning urine if possible, or if not, limiting water intake in the hour prior to giving a sample. Repeat testing may be useful.

Confirmation tests are usually required for medicolegal purposes when drug testing is used in the workplace or for family custody disputes in which parents are allegedly using drugs at home in the presence of children. An initial urinary screening test must be confirmed for evidence of drug use. Confirmatory testing is more sensitive and specific than screening tests and confirms the drug of interest as opposed to the drug class.

IA is non-specific and cross-reactions can occur. As an example, quinolone antibiotics can cross react with an opioid panel yielding a false 
positive test result. To eliminate this type oferror, IA tests should be confirmed with a more definitive chromatographic test (e.g., GCMS), particularly if a test result is unexpected and does not correlate with a patient's history.

The evolution of oral-fluid testing (saliva) both from a policy and technology viewpoint has grown rapidly in recent years. This testing has been used primarily to test drivers for illicit drugs (amphetamines and cannabis) and is now being used in the workplace.

Saliva is easier and safer to collect than urine. Unlike urine, not all drugs are easily detectable in oral fluid, either because of the low concentrations or the short time that drugs are present in saliva. The detection of a drug in oral fluid is normally associated with recent use (up to 24 hours) as the drugs can be related to blood or plasma concentrations and therefore physiological effect. This cannot be inferred from urine as the concentration in urine can only be related to previous consumption and not any effect of the drug.

\section{Conclusion}

Adolescents need to be treated as a distinct segment of our population and it is important to realize and address their health and lifestyle problems. Inadequate sleep, depression and smoking were the leading unhealthy behaviours among the respondents. Families can play an important role to help these adolescents live a healthier life. Because substance-use disorders are common, UDT should be considered a core tool in primary care. The clinician can use a discordant UDT result to motivate patient behavior change. However, testing without an appropriate strategy for interpreting results can do harm. A working relationship with the testing laboratory or test kit provider is essential to accurately interpret UDT results. Most importantly, a health care professional should strive for a relationship of mutual honesty and trust with the patient when using UDT in his or her clinical practice. Therefore, the use of UDT should be consensual, be designed to help patients, and assist health care professionals to advocate for the health and well-being of their patients.

\section{Acknowledgement}

The authors are thankful to IJIST Journal for publishing their article.

\section{Conflicts of Interest}

The author declares that there are no conflicts of interest.

\section{References}

1. Borrmann, S., S. Issifou, G. Esser, A. A. Adegnika, M. Ramharter, P. B. Matsiegui, S. Oyakhirome, D. P. Mawili-Mboumba, M. A. Missinou, J. F. Kun, H. Jomaa, and P. G. Kremsner. Fosmidomycinclindamycin for the treatment of Plasmodium falciparum malaria. J. Infect. Dis. 2004; 190:15341540.Abstract/FREE Full Text

2. Boyle, J. The effect of the high school on students' aspirations. Amer .J. Sociol., 71, 628-639.

3. McConkey, G. A., M. J. Rogers, and T. F. McCutchan. Inhibition of Plasmodium falciparum protein synthesis. Targeting the plastid-like organelle with thiostrepton. J. Biol. Chem. 1997; 272:20462049.Abstract/FREE Full Text

4. Medline GustafssonL. L., et al. Disposition of chloroquine in man after single intravenous and oral doses. Br. J. Clin. Pharmacol. 1983; 15:471479.2006.

5. Meshnick, S. R., Taylor, T. E. \& Kamchonwongpaisan, S. Artemisinin and the antimalarial endoperoxides: from herbal remedy to targeted chemotherapy. Microbiol. Rev. 1996; 60:301-315.

6. Roos, V., Nielsen, E.M. and Klemm, P. Asymptomatic bacteriuria Escherichia coli strains: adhesins, growth and competition. FEMS Microbiol Lett 2006; 262:22-30.

7. Rosenberger, C.M. and Finlay, B.B. Phagocyte sabotage: disruption of macrophagesignalling by bacterial pathogens. Nature reviews. Molecular cell biology 2003; 4:385-396.

8. Foth, B. J., S. A. Ralph, C. J. Tonkin, N. S. Struck, M. Fraunholz, D. S. Roos, A. F. Cowman, and G. I. McFadden.. Dissecting apicoplast targeting in the malaria parasite Plasmodium falciparum. Science 2003; 299:705-708.Abstract/FREE Full Text.

9. Frisk-HolmbergM., BergqvistY., TermondE., Domeij-NybergB. The single dose kinetics of chloroquine and its major metabolite desethylchloroquine in healthy subjects. Eur. J. Clin. Pharmacol. 1984; 26:521-530.CrossRefMedline.

10. Cook, J. A., E. J. Randinitis, C. R. Bramson, and D. L. Wesche. Lack of a pharmacokinetic interaction between azithromycin and chloroquine. Am. J. Trop. Med. Hyg. 2006; 74:407-412.Abstract/FREE Full Text.

11. Cox-Singh, J. et al. Plasmodium knowlesi malaria in humans is widely distributed and potentially life threatening. Clin. Infect. Dis. 2008; 46:165-171.

12. Weissig, V., T. S. Vetro-Widenhouse, and T. C. Rowe. Topoisomerase II inhibitors induce cleavage 
of nuclear and $35-\mathrm{kb}$ plastid DNAs in the malarial parasite Plasmodium falciparum. DNA Cell Biol. 1997; 16:1483-1492.
13. Cassidy N, Herbert JX, Tracey JA. The availability of toxicological analyses for poisoned patients in Ireland. Clin Toxicol 2010; 48:373-9. 